Mots. Les langages du politique

104 | 2014

Les livres de journalistes politiques

\title{
Langue et politique, passion française
}

Language and politics, a French passion

Pierre Fiala

\section{Q OpenEdition}

Journals

Édition électronique

URL : https://journals.openedition.org/mots/21639

DOI : $10.4000 /$ mots. 21639

ISSN : 1960-6001

\section{Éditeur}

ENS Éditions

\section{Édition imprimée}

Date de publication : 19 mai 2014

Pagination : 107-117

ISBN : 978-2-84788-542-2

ISSN : 0243-6450

\section{Référence électronique}

Pierre Fiala, «Langue et politique, passion française », Mots. Les langages du politique [En ligne], 104 | 2014, mis en ligne le 19 mai 2016, consulté le 23 avril 2022. URL : http://journals.openedition.org/ mots/21639; DOI : https://doi.org/10.4000/mots.21639

\section{(c) ENS Éditions}




\author{
Pierre Fiala
}

\title{
Langue et politique, passion française
}

Politique et langage ${ }^{1}$, éléments constitutifs du lien social et de sa conflictualité, sont particulièrement indissociables dans la société française. Ils sont omniprésents et quasiment inséparables dans l'espace public : débats institutionnels, juridiques, législatifs sur fond de définitions et de dénominations controversées ${ }^{2}$, joutes oratoires parlementaires ou partisanes, polémiques médiatiques présentées comme performances sportives ou théâtrales où la forme et le succès l'emportent sur le fond et les principes, événements discursifs construits par les agendas de communicants, mises en scène répétées d' "éléments de langage », d'effets d'annonce, actes de langages en tout genre, petites phrases, qualificatifs plus ou moins insultants aux conséquences judiciaires diverses33, commentaires médiatiques continus4, échanges amplifiés dans les réseaux numériques sociaux, conduisant fréquemment à des

* Ce texte constitue la partie initiale d'une contribution de Ronny Scholz et Pierre Fiala, «Sprache und Politik im Frankreich », à paraître en 2015 dans Vorläufige Gliederung des geplanten Handbuchs, Sprache und Politik, J. Kilian, T. Niehr, M. Wengeler éd.

1. Là où l'allemand et l'anglais utilisent un seul mot, le français, comme d'autres langues romanes (italien, espagnol), distingue deux termes, langue et langage, qui ont accentué sinon fondé une distinction conceptuelle entre la faculté de langage, extensive, et l'objet langue, défini de façon structurelle. Cette distinction qui marque la réflexion théorique francophone est, on le sait, une source de difficulté pour les traducteurs.

2. Les exemples ne manquent pas: insertion, assimilation, intégration, limitation, problème, seuil de tolérance, etc., autant de maîtres-mots qui ont marqué les différentes politiques d'immigration. Remplacement du terme juridique connoté inculpation par l'expression descriptive mise en examen; du terme probatoire par le terme contrainte, jugé moins laxiste; nouvelle terminologie pour le Code civil instaurant le mariage sans distinction de sexe, nommé mariage pour tous.

3. L'extrême droite et les dirigeants du Front national en ont été les pourvoyeurs principaux depuis trente ans.

4. Le terme technique sémantique s'est généralisé, souvent abusivement, dans les débats actuels et les commentaires politiques pour caractériser les arguments à caractère linguistique. Voir l'article intitulé « Marine Le Pen tente une bataille sémantique» (Le Monde, 5 octobre 2013), qui relate les tentatives répétées du Front national visant à empêcher que lui soit appliqué le qualificatif d'extrême droite, y compris par des moyens judiciaires, utilisant pour sa part l'expression «guerre sémantique».

CEDITEC, EA 3119, Université Paris Est Créteil Val-de-Marne pfiala@noos.fr 
violences verbales qui portent sur les normes de langue, la légitimité, la bienséance, enquêtes d'opinion alimentant des controverses et des polémiques quasiment permanentes 5 , titres et chroniques de presses jouant sur les mots, caricatures, imitations, humoristes associant l'image et le verbe, publications en tout genre ${ }^{6}$. Les pratiques langagières, au travail comme le domaine privé, connaissent ce même ancrage réciproque du langagier et du politique dans les conversations quotidiennes, l'échange de courriers, les vannes et les bons mots, les lectures, les messages numériques de toutes sortes remettant souvent en cause les règles de la civilité (Balibar, 2010).

Cette passion sociale du politico-langagier, constitutive de l'imaginaire national, va au-delà des concepts restreints définis par les sciences de la langue ou du politique. Nous l'abordons dans son extension large, langagière, sociohistorique et pratique en suivant, d'une part, dans la durée les domaines de connaissance, souvent interdisciplinaires7, qui ont cherché à la théoriser avec des méthodes spécifiques et en replaçant, d'autre part, l'activité scientifique dans le cadre social et politique dont on ne peut ici moins qu'ailleurs faire abstraction. Du point de vue langagier qui est le nôtre, nous nous efforçons de répondre dans un premier temps à la question suivante : Quelles sont les origines historiques des recherches articulant langage et politique? D'autres questions en découlent qui pourront être abordées plus tard : Dans quelles conditions apparaît, dans les années soixante / soixante-dix, l'analyse du discours politique française (désormais ADP), qui approfondit cette relation dans le contexte intellectuel de critique sociale et politique de l'époque? Quels sont les liens et les différences entre l'ADP et les autres traditions de recherche nom-

5. Parmi les débats récurrents récents, entretenus par les médias et mobilisant la société audelà des groupes institutionnels et des partis, on peut noter ceux sur les identités sociales, le sexisme, le genre et le mariage pour tous; le voile islamique, l'islamophobie et la laïcité; les ressources énergétiques et l'écologie politique; plus anciens mais toujours présents, l'antisémitisme récurrent, la sécurité, la violence urbaine, les étrangers et le racisme; les inégalités économiques et sociales, les réformes institutionnelles, de la santé, de l'éducation, des divers enseignements, de l'orthographe. Une étude détaillée de la parole sociopolitique circulante ferait apparaître sans doute une certaine baisse d'intensité du débat traditionnel et de l'investissement citoyen, ainsi qu'un déplacement des controverses vers les réseaux sociaux numériques et vers des débats plus minoritaires et plus violents.

6. Livres politiques, biographies, essais divers, brochures, pamphlets, dictionnaires des divers parlers à la mode, de la langue de bois, de la langue de mousse, du parler parisien, etc.

7. Il ne sera question qu'indirectement du vaste domaine théorique et d'intervention appelé politique linguistique (ou glottopolitique) qui désigne l'aménagement et la gestion par l'État de la langue française, de son statut national et international, de la francophonie, de la variété et des langues minoritaires de l'hexagone, des normes terminologiques, grammaticales, orthographiques, du système éducatif. Voir notamment à ce sujet Balibar et Laporte (1974) qui en ont étudié les origines (voir Branca éd., 2001), De Certeau et al. (1975), qui analyse la politique linguistique durant la Révolution, Calvet (1974), qui analyse les effets destructeurs de la politique coloniale sur les langues colonisées ; Vermès et Boutet (1987), qui ont souligné l'autonomie paradoxale entre les mesures politiques en France et les recherches effectives en sociolinguistique. Un ouvrage récent (Kremnitz éd., 2013) sur les langues parlées en France permet d'en mesurer les progrès scientifiques mais aussi politiques. 
breuses portant sur le langage et la politique, à commencer par la lexicométrie politique, perspective propre à la recherche en France? Comment cette relation a-t-elle évolué dans les années récentes, notamment depuis l'apparition des sciences de l'information et de la communication, liées à la croissance des réseaux sociaux sur Internet, dans un contexte où la substance politique tend à s'effacer devant les techniques de communication?

La relation langage/politique est d'abord ancrée dans la tradition rhétoricogrammaticale. Elle repose sur les notions traditionnelles de discours ${ }^{8}$ et de mot9. Ces notions ont été critiquées et redéfinies au cours du xxe siècle par les linguistes structuralistes et les sociolinguistes (Boyer, 1996). Ce sont des méthodes et des disciplines récentes, comme la sociolexicologie politique, l'analyse du discours, la statistique lexicale, qui ont particulièrement exploré et illustré la relation entre la langue et le politique durant ces dernières décennies. La réflexion portera en premier lieu sur ces domaines, sans négliger les travaux qui traitent des rapports entre langage et politique dans des perspectives voisines, historiques, sociologiques ${ }^{10}$, politologiques, philosophiques. On s'efforcera ainsi de mettre en lumière, dans la durée et dans ses aspects interdisciplinaires, la dimension politique du langagier et la dimension linguistique du politique. L'ambition de ce point de vue n'est ni réaliste ni raisonnable; sa seule justification est qu'elle correspond en fait à une expérience individuelle d'une cinquantaine d'années passées à tenter d'articuler des savoirs et des engagements.

\section{Quelques repères pour une lexicologie politico-historique dans la durée}

Dans le sillage des premiers penseurs européens, italiens (Machiavel, Guichardin) et anglais (Bacon, Hobbes, Locke en particulier, avant Hume), la pensée politique connaît dans la France des Lumières un développement considérable. Montesquieu, Voltaire, Mably, Raynal, forgent les outils fondamentaux de la pensée et de l'action politique, avec une référence privilégiée au langage ${ }^{11}$.

8. Le terme discours, employé anciennement avec une valeur extensive englobant des manifestations multiples de la parole, s'est spécialisé à l'âge classique pour désigner l'expression verbale, écrite ou orale, de la pensée maîtrisée par la raison et/ou l'art oratoire. La linguistique structurale a précisé, d'une part, la définition du discours en le liant à l'ordre de langue et aux conditions de l'énonciation et a, d'autre part, élargi son extension aux diverses réalisations de la parole, prises pour objets d'observation par les sciences humaines et sociales.

9. Voir Branca (1998) sur la notion de mot.

10. Par exemple l'approche sociologique du « marché linguistique » de Bourdieu, les travaux sociolinguistiques de Calvet $(1974,1977,1995)$ ancrés dans le politique, ceux de Guilhaumou (1998) ou de Boutet (2010) qui s'inscrivent comme des interventions scientifiques dans l'actualité politique et sociale.

11. Guilhaumou (2006) en indique les moments principaux dans le chapitre «L'histoire des événements linguistiques. Le cas de la “Langue française” au xvıı' siècle». Voir aussi Auroux, 1986. 
Rousseau ${ }^{12}$ surtout inspire les acteurs et théoriciens de la Révolution, de Robespierre à Siéyès ${ }^{13}$, Condorcet, l'abbé Grégoire. Formés par la rhétorique classique, ces penseurs font du langage, où s'articule raison, passion et action, l'instrument privilégié de la persuasion politique, de l'éloquence d'assemblée, de la construction effective du pouvoir nouveau, mais aussi et surtout des théories qui le fondent. Ils réfléchissent sur le pouvoir des mots, leur abus ${ }^{14}$, la puissance effective des discours, des devises, des titres et des chants, des formules dans la mise en œuvre des fondements de la République et de la Nation ${ }^{15}$. Ils organisent la parole publique, réglementent la prise de parole dans le débat parlementaire (Brasart, 1988), créent les terminologies propres à la pensée et à l'ordre républicains ${ }^{16}$, mettent en place une politique linguistique nationale, voire universaliste ${ }^{17}$, où le langage devient le lieu d'événements, de luttes idéologiques ${ }^{18}$ et d'élaboration politique ${ }^{19}$, non sans une certaine ostentation liée à la pratique de l'éloquence.

Le lien étroit entre langage et politique se perpétue, dans la tradition philosophique, historique et littéraire du xıxe siècle, tant dans la pensée réactionnaire des idéologues (de Bonald, Joseph de Maistre) que dans la pensée libérale (de Benjamin Constant à Tocqueville) en passant par les grandes narra-

12. Discours sur l'origine et les fondements de l'inégalité parmi les hommes (1755), Essai sur l'origine des langues (1761), L'Émile (1762), Le Contrat social (1762) dont un manuscrit original est conservé à l'Assemblée nationale.

13. Auteur notamment de Qu'est-ce que le Tiers-État? (1789).

14. Notion, introduite par Locke et développée par Siéyès, pour décrire d'un point de vue prescriptif les excès de l'éloquence politique.

15. Liberté, égalité, fraternité; Droits de l'homme et du citoyen; Droite et Gauche; Peuple et Nation; La patrie en danger; patriotes et émigrés; La terreur à l'ordre du jour ; Les aristocrates à la lanterne, etc.

16. Le Supplément du Dictionnaire de l'Académie de 1798 réunit les néologismes techniques, scientifiques et politiques qui fondèrent des tentatives éphémères ou durables de transformations sociales et politiques (nouveau calendrier, système métrique, institutions étatiques). Les dictionnaires, dès l'Ancien Régime et la création de l'Académie française (1636) sont en France un outil d'organisation politique et idéologique, qu'il s'agisse de la visée unificatrice des éditions successives du Dictionnaire de l'Académie, de la visée religieuse et morale des rééditions du dictionnaire de Furetière (1694) par les jésuites de Trévoux ou de la vaste entreprise progressiste de l'Encyclopédie de Diderot et d'Alembert (1751-1772).

17. L'abbé Grégoire, en réalisant son enquête sur les patois, jette les bases d'une politique républicaine de centralisation linguistique dans le prolongement de l'unification politico-linguistique entreprise dès l'Ancien Régime, sur des bases parfois aberrantes dont le Discours sur l'universalité de la langue française de Rivarol (1784) est un exemple. Voir Balibar et Laporte (1974), De Certeau et al. (1975), Auroux (1986).

18. Les huit volumes (1985-2006) du Dictionnaire des usages sociopolitiques (1770-1815) proposent des analyses approfondies des mots fondateurs de la pensée républicaine.

19. Des historiens contemporains (Koselleck, 1997) se sont référés à la période de 1750 à 1850 sous le terme Sattelzeit, qui voit l'élaboration en Europe des fondements de diverses théories politiques de la liberté où la Révolution française joue un rôle pivot essentiel. On notera toutefois une différence dans la tradition allemande plus conceptuelle, délibérative, ancrée dès son origine dans la réflexion logico-juridique autour de Leibniz, Grotius, Pufendorf. Cette tradition réflexive qui se prolonge à travers les concepts fondateurs du matérialisme historique et de l'économie politique de Marx se retrouve dans la pensée contemporaine de Weber à Habermas, ou dans le Dictionnaire des concepts sociopolitiques (1972-1997). 
tions, qu'elles soient conservatrices (Chateaubriand, Taine, Thiers) ou progressistes et républicaines (Michelet, Saint-Simon, Comte, Hugo). Le positivisme contribue à approfondir l'héritage du langage révolutionnaire et républicain face aux divers courants monarchistes ou bonapartistes ${ }^{20}$. Avec le dictionnaire de Littré (1862-1873) et les dictionnaires encyclopédiques de Larousse (18641876), la lexicographie moderne naissante fonde dans la science des mots euxmêmes, l'émancipation du peuple, les nouveaux droits politiques, le progrès social, les bases de la laïcité, de l'école républicaine et de la démocratie républicaine, qui seront brandis dans les crises sociales et politiques du siècle ${ }^{21}$.

\section{L’ancrage dans l'histoire et la linguistique}

Liée encore fortement à la littérature22, l'articulation du politique et du langagier au début du xxe siècle prend une tournure théorique et méthodologique, tant chez les historiens que dans les sciences du langage en plein développement, alimentée d'abord par des travaux lexicologiques dont le plus remarquable est l'histoire monumentale de la langue française de Ferdinand Brunot 23 . Dans sa conception progressiste de la langue conçue comme outil de l'unification nationale et républicaine, Brunot écrit en 1913 (préface du tome IV) : «La linguistique actuelle [...] se tourne de plus en plus vers l'histoire pour former une branche de l'histoire sociale », et dans l'introduction du tome VII, il précise :

L'histoire de la langue s'enrichirait de toutes les données que peut fournir l'histoire générale, et inversement, elle contribuerait à rendre celle-ci plus humaine et plus profonde, puisque la langue est le fait social par excellence, qu'elle se modifie sans doute par les volontés individuelles, mais ne cesse jamais d'être un produit de la collectivité et reflète par conséquent, avec une fidélité unique, l'état des esprits, des mœurs, de la vie aux différentes époques. (Brunot, 1926, p. 4-5)

20. Dans le premier chapitre de l'Histoire de la Révolution française, Michelet analyse dans le détail le choix des mots et des usages nouveaux (Tiers-État; Députés, Peuple, Nation), des phrases clés, des actes de langage par lesquels le mouvement révolutionnaire réduit le pouvoir de l'Ancien Régime dans les premiers mois de 1789 et instaure le nom et le pouvoir de l'Assemblée nationale. On trouve là un modèle de pragmatique lexico-politique avant la lettre.

21. Paul Lafarge décrit «La langue française, avant et après la Révolution » (L’Ėre nouvelle, 1894, republié dans Calvet, 1977). Émile Zola, quant à lui, dans J'accuse (1898), mobilise les outils rhétoriques pour rétablir la vérité et l'honneur du capitaine Dreyfus et dénoncer l'antisémitisme régnant dans l'armée et les institutions. On notera l'importance de la tradition anarchiste (Leroux, Proudhon, Saint-Simon) durant tout le siècle. Angenot (2005) montre par ailleurs l'influence considérable des traductions des œuvres de Marx et Engels à la fin du siècle.

22. La première guerre mondiale fait l'objet, on le sait, de nombreuses réflexions lexicales chez Proust (À la Recherche du temps perdu, 1913-1927) ou chez Barbusse (Le Feu, 1916).

23. Dans l'Histoire de la langue française en 21 volumes, Brunot (1860-1938) propose une approche historique extensive (externe et interne) de la grammaire, du vocabulaire (général, technique, philosophique, littéraire) et notamment du vocabulaire sociopolitique (xvIII siècle et Révolution). On trouve dans les derniers volumes consacrés au xxe siècle d'importantes contributions sur le vocabulaire politique. Voir Tournier et al. (1995). 
En écho, un historien comme Lucien Febvre 24 s'intéresse de près au vocabulaire et aux usages sémantiques 25 . On retrouve chez lui mot pour mot la position de Brunot : "La langue étant le fait social par excellence reflète avec une fidélité unique l'état général de la civilisation aux diverses époques » ${ }^{26}$. II écrit à propos de Brunot et Meillet :

Il faut la collaboration de ces «sémantistes» qui, en nous restituant l'histoire de mots particulièrement lourds de sens, écrivent du même coup des chapitres précis d'histoire des idées. Il faut la collaboration de ces historiens des langues - tel Meillet décrivant l'histoire de la langue grecque, tel Ferdinand Brunot suivant pas à pas les destinées de la langue française. ${ }^{27}$

Initiés par Brunot et Lucien Febvre, les fondements d'une lexicologie sociohistorique orientée politiquement vers le progrès social et le développement démocratique se mettent en place entre 1925 et 1940. La démarche reste tributaire de la démarche lexicographique, souvent liée aux textes littéraires ${ }^{28}$, éloignée encore des problématiques discursives, de la constitution de corpus textuels, de l'utilisation des méthodes linguistiques. Ces préoccupations fondent en revanche les travaux comparatistes de Meillet puis de Benveniste ${ }^{29}$. Elles prendront de l'ampleur dans les approches sociales et politiques du vocabulaire de Dubois (1962), dans une perspective sociolexicologique reposant sur l'érudition philologique et historique mais également sur les acquis de la linguistique structurale. Analysant par exemple le mot égalité, Dubois (1962) met en lumière son passage du vocabulaire général au lexique proprement politique dans le courant du XVIIIe siècle (p.78). Il note que la Révolution adjoint à la notion d' «égalité politique » celle d' "égalité sociale» et rappelle, avec Pierre Leroux, que le mot résume «tous les progrès antérieurs accomplis jusqu'ici par l'Humanité » (De l'Égalité, 1838), ajoutant que si «inégalité était autrefois synonyme d'homme, aujourd'hui c'est égalité qui est ce synonyme »(De l'Humanité, 1840). Dubois souligne «la grande résonance affective » du terme en 1789 , qui se retrouve à la fin de l'Empire. Le terme faisant partie de la phraséologie révolutionnaire, il est dénoncé par la réaction : «On a jeté aux quatre vents du ciel ces mots vides et sonores : Plus de castes! Plus de privilèges! Plus de distinction de rang, de titres, de fortune

24. Fondateur avec Marc Bloch de l’École des Annales qui, entre autres, introduit dans la méthode historique une conception globale et interdisciplinaire fondée sur la longue durée, la recherche des structures des faits sociaux et la collaboration étroite entre disciplines.

25. Auteur de plusieurs études lexicales : «Frontière : Le mot et la notion » (1928), article qui s'inscrit dans une démarche de type structural, systématique, reposant sur les dictionnaires, de type onomasiologique, répondant à un plan fixe; «Civilisation : évolution d'un mot et d'un groupe d'idées» (1929), article qui donnera lieu à de nombreux développements ; « Capitalisme et capitaliste : mots et choses» (1939); «Travail : évolution d'un mot et d'une idée» (1941).

26. Revue de synthèse historique, 1926 ; repris dans Combats pour l'histoire, 1992.

27. "Histoire et psychologie », 1938 ; repris dans Combats pour l'histoire, 1992, p. 218.

28. Matoré (1951) étudie le vocabulaire social de 1848 à partir des textes littéraires de l'époque. Tournier (1975) abordera la même période à partir de l'analyse du vocabulaire ouvrier.

29. Voir le Vocabulaire des institutions indo-européennes (1969). 
et d'honneurs! L’Égalité parfaite! L’Égalité pour tous et en toutes choses! » écrit l'abbé Bournichon (cité par Vermorel, 1870). Le terme résonne aussi dans l'écriture littéraire, dans L'Éducation sentimentale (1869) : «Le répétiteur (Sénécal) avait été congédié de son troisième pensionnat pour n'avoir point voulu de distribution de prix, usage qu'il regardait comme funeste à l'égalité [...] Nous ne voulons pas d'aumônes, entendez-vous! Mais l'égalité, la juste répartition des produits », et Flaubert souligne lui-même dans une lettre à Georges Sand (corresp. IV, 80) : «L'idée d'égalité (qui est toute la démocratie moderne) est une idée essentiellement chrétienne et qui s'oppose à celle de justice. » Jean Dubois souligne enfin sur le plan politique que "La Commune précise de quelle sorte d'égalité elle demande l'instauration »: «l'égalité sociale» (dans le Journal officiel de la Commune), «la liberté politique par l'égalité sociale», selon les candidats socialistes de l'époque, qui doit permettre «l'avènement politique des travailleurs ». C'est de «l'égalité civile», fondement de ce siècle, que doit procéder cette "égalité sociale». Après avoir analysé les contextes du terme, l'auteur en analyse les propriétés morphologiques, étudiant la série étymologique à valeur politique dérivée du terme, notamment durant la Révolution : égalisation ou égalisation des classes avec le sens de «nivellement des classes sociales». De inégalité on a tiré en 1839 inégaliser. On retrouve alors les emplois néologiques de niveler, niveleur, nivellement, voire aplanisseur, apparus au moment de la Révolution. Mais la formation la plus caractéristique de l'intégration de égalité dans le champ politique et social est la dérivation de l'adjectif qualificatif égalitaire, attesté dès 1840 dans la langue socialiste. Selon Dezamy (1840, Premier banquet communiste), «les communistes sont une "École égalitaire" ». Blanqui s'en revendique. Le mot est fréquent sous la Commune. Apparaissent alors les compositions anti-égalitaire, inégalitaire et égalitarisme dans son analyse du mot égalité dont les valeurs sont dégagées à partir de leur contexte. Arnould (1870) souhaite une «société égalitaire» tandis que l'ecclésiastique Veuillot et d'autres ironisent surces « discours égalitaires», cette « démocratie égalitaire», les «rêves égalitaires».

La perspective sémantique et historique de la lexicologie a ainsi participé aux débats historiographiques ${ }^{\circ}$. Elle a été prise en compte par les sciences politiques ${ }^{31}$. Alimentée par des données nouvelles, elle a pris dans les dernières décennies une dimension anthropologique. Des monographies approfondies restituent la profondeur historique des mots et de leur famille dans leurs usages sociaux, dans la durée, par exemple la construction du mot et du concept de classe aux XVIIII et XIXe siècles (Piguet, 1996), ou du mot révolution

30. Voir par exemple les interprétations politiques profondément divergentes de la Révolution française par les historiens Soboul, Vovelle ou Furet.

31. Rémont (1982) souligne l'apport nécessaire des approches lexicologiques, quantitatives notamment, à la science politique. Voir aussi Bacot (2011). 
(Rey, 1989). Les travaux collectifs sur les usages sociolexicaux32 et les pratiques langagières (Branca, Schneider, 1994) sous la Révolution, sur les usages et les variations discursives des mots de l'égalité et de l'inégalité (Fiala et al., 1999), sur les mots du syndicalisme ouvrier33 et les nombreux travaux d' «étymologie sociale» de Maurice Tournier sur les mots de la grève, les mots du politique, les mots de Mai 1968, les noms de la République34, proposent des synthèses et constituent aujourd'hui des références méthodologiques.

Illustrée par ces travaux nombreux, la lexicologie socio-historico-politique s'est constituée comme un ensemble de méthodes, distinctes de la lexicographie traditionnelle, associant les acquis de la philologie historique, le recueil archivistique de matériaux textuels divers, couvrant l'espace public au-delà de l'espace politique (jusqu'à la langue parlée d'un côté, la littérature de l'autre), détaillant la mise en forme de relations structurales, morphologiques (dérivations, composition, phraséologie) et sémantiques (synonymies, antonymies, métaphorisation, etc.) à travers des tests linguistiques; relevant des usages dans différents contextes grammaticaux; l'interprétation des valeurs par comparaison des usages en discours et de leur évolution historique et sociale. Elle constitue aussi le substrat méthodologique, thématique et idéologique sur lequel s'est développée l'ADP en France. Centrée, directement ou indirectement, sur des questionnements politiques, celle-ci vise une objectivité propre à la méthodologie scientifique, sans pour autant se retrancher dans une neutralité détachée des enjeux sociaux et politiques de son objet. C'est un autre épisode.

\section{Références}

ANGENOT Marc, 2006, Le marxisme dans les grands récits. Essai d'analyse du discours, Laval, Paris, L'Harmattan.

Arnold Nicole, Dougnac François, Gefrroy Annie, Guilhaumou Jacques, Monnier Raymonde, PIgUet Marie-France et al., 1985-2006, Dictionnaire des usages sociopolitiques (1770-1815), 8 vol., Paris, Champion.

Auroux Sylvain, 1986, «Le sujet de la langue. La conception politique de la langue sous l’Ancien Régime et la Révolution », Les Idéologues, W. Busse, J. Trabant, Amsterdam, Benjamins, p. 259-278.

BALIBAR Étienne, 2010, Violence et civilité. Wellek Library Lectures et autres essais de philosophie politique, Paris, Galilée.

32. Dictionnaire des usages sociopolitiques, 1750-1815.

33. Voir les travaux de Tournier (1975) sur le vocabulaire des pétitions ouvrières de 1848 , et les études collectives systématiques du vocabulaire syndical ouvrier au xxe siècle. Voir Hetzel et al. (1998).

34. Tournier $(1975,1992,1995,2002,2007)$ a défendu et illustré l'idée que la forme des mots porte toujours des traces de l'histoire sociale de leurs usages. S'appuyant sur des principes bakhtiniens, le lexicologue, à l'instar du préhistorien ou de l'archéologue, et parfois du poète, peut ainsi, en étudiant l'évolution de la forme des mots et de leur famille, leur morphologie, faire resurgir des mondes engloutis. 
Balibar Renée, 1993, Le colinguisme, Paris, PUF (Que sais-je).

BAlibAR Renée, LAPORTE Dominique, 1974, Le français national. Politique et pratique de la langue française sous la Révolution, Paris, Hachette.

BACOT Paul, 2011, La construction verbale du politique, Paris, L'Harmattan.

BAcot Paul et al., 2010, Trente ans d'étude des langages du politique (1980-2010), $\mathrm{n}^{\circ} 94$ de Mots. Les langages du politique, novembre 2010.

Benveniste Émile, 1969, Vocabulaire des institutions indo-européennes, Paris, Minuit.

Bonnafous Simone, Chiron Pierre, Ducard Dominique, Lévy Carlos éd., 2003, Argumentation et discours politique. Antiquité grecque, latine, Révolution française, Monde contemporain, Rennes, Presses universitaires de Rennes.

Boutet Josiane, 2010, Le pouvoir des mots, Paris, La Fabrique.

BOUTET Josiane, VARRO Gabrielle éd., 2007, La revue a 30 ans. Réflexions et perspectives de recherche, $\mathrm{n}^{0}$ 121-122 de Langage \& société, septembre-décembre 2007.

BOYER Henri éd., 1996, Sociolinguistique. Territoire et objets, Lausanne, Delachaux et Niestlé.

BrancA-Rosoff Sonia éd., 1998, Le Mot. Analyse du discours et sciences sociales, Aixen-Provence, Publications de l'Université de Provence.

- 2001, L'institution des langues. Autour de Renée Balibar, Paris, Maison des sciences de l'homme.

Branca-Rosoff Sonia, SchneIder Nathalie, 1994, L'écriture des citoyens. Une analyse linguistique de l'écriture des peu lettrés pendant la Révolution française, Paris, Klincksieck.

Brasart Patrick, 1988, Paroles de la Révolution. Les Assemblées parlementaires 17891794, Paris, Minerve.

- 1994, " "Petites phrases et grands discours”. Sur quelques problèmes de l'écoute du genre délibératif sous la Révolution française», Mots, n 40, 1994, p. 106-112.

BRUNOT Ferdinand, 1905-1938, Histoire de la langue française, des origines à 1900, 21 vol. (dont 11 publiés du vivant de l'auteur; trois volume supplémentaires : 1870$1914 ; 1914-1945 ; 1945-2000)$, Paris, Armand Colin.

CAlvet Louis-Jean, 1974, Linguistique et colonialisme. Petit traité de glottophagie, Paris, Payot.

- 1977, Marxisme et linguistique, Paris, Payot.

- 1995, Les politiques linguistiques, Paris, PUF (Que sais-je).

Charaudeau Patrick, 2005, Le discours politique. Les masques du pouvoir, Paris, Vuibert.

- 2013, La conquête du pouvoir. Opinion, persuasion, valeur. Les discours d'une nouvelle donne politique, Paris, L'Harmattan.

De Certeau Michel, Julia Dominique, ReVel Jacques, 1975, Une politique de la langue. La Révolution française et les patois, Paris, Gallimard.

Deleplace M., 2000, L'anarchie de Mably à Proudhon (1750-1850). Histoire d'une appropriation polémique, Lyon, ENS Éditions.

DENQUIN Jean-Marie, 1997, Vocabulaire politique, Paris, PUF.

DuвoIs Jean, 1962, Le vocabulaire politique et social en France de 1869 à 1872. À travers les œuvres des écrivains, les revues et les journaux, Paris, Larousse.

FebVRe Lucien, 1992, Combats pour l'histoire, Paris, Armand Colin. 
FIALA Pierre éd., 1999, In/égalité/s. Usages lexicaux et variations discursives (XVIII xxe siècles), Paris, L'Harmattan.

Guilhaumou Jacques, 1989, La langue politique et la Révolution française. De l'événement à la raison linguistique, Paris, Méridiens/Klincksieck (trad. allemande : Sprache und Politik in der Französischen Revolution, Suhrkamp).

- 2006, Discours et événement. L'histoire langagière des concepts, Besançon, Presses universitaires de Franche-Comté.

- 1998, La parole des sans. Les mouvements actuels à l'épreuve de la Révolution française, Fontenay-aux-Roses, ENS Éditions.

Guilhaumou Jacques, Schepens Philippe éd., 2011, Matériaux philosophiques pour l'analyse du discours, Besançon, Presses universitaires de Franche-Comté.

HetzelAnne-Marie, Josette LefÈvre, René Mouriaux, Maurice Tournier, 1998, Le syndicalisme à mots découverts. Dictionnaire de fréquences (1971-1990), Paris, Syllepse.

KOSELLECK Reinhart, 1997, L'expérience de l'histoire [trad. franç.], Paris, Gallimard / Le Seuil.

KREMNITZ Georg éd., 2013, Histoire sociale des langues de France, Rennes, Presses universitaires de Rennes.

LAUNAY Michel, 1972, Le vocabulaire politique de Jean-Jacques Rousseau, Genève, Paris, Slatkine, Champion.

MATORÉ Georges, 1951, Le Vocabulaire et la société sous Louis-Philippe, Genève, Droz.

MulLer Pierre, 1994, Jaurès, vocabulaire et rhétorique, Paris, Klincksieck.

Piguet Marie-France, 1996, Classe. Histoire du mot et genèse du concept, des Physiocrates aux Historiens de la Restauration, Lyon, Presses universitaires de Lyon.

RANCIÈRE Jacques, 1998, Au bord du politique, Paris, La Fabrique.

RÉMI-GIRAUd Sylvianne, RÉTAT Pierre éd., 1996, Les mots de la nation, Lyon, Presses universitaires de Lyon.

RÉMONT René, 1982 [1954], Les Droites en France de 1815 à nos jours, Paris, Aubier-Montaigne ( 4 é édition).

RENNES Juliette, 2007, Le mérite et la nature. Une controverse républicaine : l'accès des femmes aux professions de prestige, 1880-1940, Paris, Fayard.

ReY Alain, 1989, «Révolution ». Histoire d'un mot, Paris, Gallimard.

SCHEPENS Philippe éd., 2006, Catégories pour l'analyse du discours politique, $\mathrm{n}^{\circ} 21 \mathrm{de}$ Semen, avril 2006.

SCHLiebEN-LANG B Brigitte, 1996, Idéologie, révolution et uniformité de la langue, Liège, Mardaga.

TOURNIER Maurice, 1975, Un vocabulaire ouvrier en 1848. Essai de lexicométrie, Quatre volumes multicopiés, Saint-Cloud, ENS.

- 1992, Les mots sur la grève. Propos d'étymologie sociale, vol. I, Paris, Klincksieck.

- 1997, Les mots en politique. Propos d'étymologie sociale, vol. II, Paris, Klincksieck.

- 2002, Des sources du sens. Propos d'étymologie sociale, vol. III, Lyon, ENS Éditions.

- 2007, Les mots de Mai 68, Toulouse, Presses universitaires du Mirail.

Tournier Maurice et al., 1975, Des tracts en mai 1968, Paris, Colin, (rééd. 1978, Paris, Champ Libre).

- 1995, "Chronique lexicale des événements politiques », Histoire de la langue française 1914-1945, Paris, CNRS, p. 207-267. 
- 2010, Des noms et des gens en République (1879-1914), Paris, L'Harmattan.

- 2013, Des mots en guerre I (1914-1939) et Des mots en guerre II (1939-1945), Paris, L'Harmattan.

VERMÈs Geneviève, Boutet Josiane éd., 1987, France, pays multilingue, I. Les langues en France, un enjeu historique et social, II. Pratiques des langues en France, Paris, L'Harmattan.

Vovelle Michel, 2004, Les mots de la Révolution, Toulouse, Presses universitaires du Mirail.

WaHNICH Sophie, 1996, L'impossible citoyen. L'étranger dans le discours de la Révolution française, Paris, Albin Michel. 